\title{
The use of virtual reality-based therapy to augment poststroke upper limb recovery
}

\author{
Geoffrey S $\underline{\text { Samuel }}^{1}$, MBBS, MRCP, Min $\underline{C h o o}^{2}$, BEng, MSc, Wai Yin $\underline{\text { Chan }}{ }^{3}$, MSc, Stanley $\underline{K o k}^{4}$, PhD, Yee Sien $\underline{\operatorname{Ng}}{ }^{1}, \mathrm{MBBS}, \mathrm{MRCP}$
}

\begin{abstract}
Stroke remains one of the major causes of disability worldwide. This case report illustrates the complementary use of biomechanical and kinematic in-game markers, in addition to standard clinical outcomes, to comprehensively assess and track a patient's disabilities. A 65-year-old patient was admitted for right-sided weakness and clinically diagnosed with acute ischaemic stroke. She participated in a short trial of standard stroke occupational therapy and physiotherapy with additional daily virtual reality (VR)-based therapy. Outcomes were tracked using kinematic data and conventional clinical assessments. Her Functional Independence Measure score improved from 87 to 113 and Fugl-Meyer motor score improved from 56 to 62 , denoting clinically significant improvement. Corresponding kinematic analysis revealed improved hand path ratios and a decrease in velocity peaks. Further research is being undertaken to elucidate the optimal type, timing, setting and duration of VR-based therapy, as well as the use of neuropharmacological adjuncts.
\end{abstract}

Keywords: kinematics, stroke, virtual reality-based therapy

\section{INTRODUCTION}

Stroke remains one of the major causes of disability worldwide.(1) Locally, cerebrovascular disease is estimated to have an incidence of 1.8 per 1000 person-years, with a crude prevalence rate of $4.05 \%{ }^{(2)}$ This case report illustrates the potential of virtual reality (VR)-based modalities for the rehabilitation of upper limb function in cases of acute stroke. We highlight the complementary use of biomechanical and kinematic in-game markers, in addition to standard clinical outcomes, to comprehensively assess and track a patient's disabilities.

\section{CASE REPORT}

A 65-year-old woman of South Indian descent was admitted to the acute stroke unit for sudden onset of right upper and lower limb weakness. The weakness was of one day's duration and was associated with an unsteady gait. The patient had a fiveyear history of hypertension, for which she was on appropriate medication. She had no significant cognitive, speech or sensory deficits. Motor power on admission was $4 / 5$ for the right shoulder, elbow, wrist, hip, knee and ankle movements and 5/5 for the left ones. Muscle tone of the affected and unaffected sides was normal. Right upper limb dysmetria was noted. She was clinically diagnosed with an ischaemic stroke and started on clopidogrel. Computed tomography on the day of admission showed chronic lacunar infarcts of the bilateral lentiform nuclei, external capsules and left centrum semiovale.

The patient was transferred to the rehabilitation medicine department on poststroke day 5. She consented to a short trial of standard stroke occupational therapy and physiotherapy with additional VR-based therapy. The VR-based therapy involved specially developed software that simulated the affected arm bringing food items to the mouth, thus encouraging elbow flexion

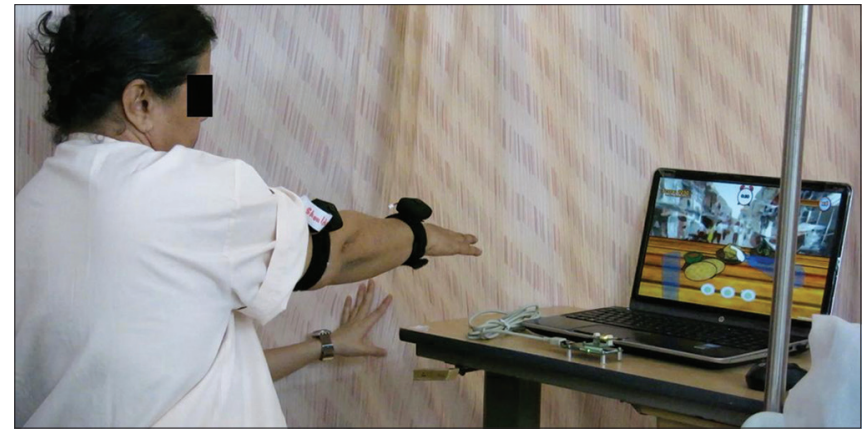

Fig. 1 Photograph shows the patient engaged in a virtual reality-based therapy session.

and extension of the arm (Fig. 1). It was conducted daily by one of the authors using a laptop computer at the patient's bedside. The patient interacted with the program via inertial measurement units (IMUs) strapped to her affected arm and wrist that detected elbow joint angle displacements and forearm trajectories. Feedback on movement quality was provided to the patient during the course of each session. After the third and final session, the patient was shown video footage of her performance during the respective session. At no point during the trial was the patient treated with neuropharmacological agents that facilitate stroke recovery.

As a measure of the smoothness of the patient's hand trajectory, (a) hand path ratios (HPR); (b) timing of flexion and extension of the affected and unaffected arms; and (c) number of velocity peaks $\left(N_{v p}\right)$ were calculated using data obtained from the IMUs. HPR is a surrogate marker for movement quality, defined as the actual length of the path traversed divided by the shortest distance between the start and end points. ${ }^{(3)}$ Thus, a path that deviates further from a straight line would result in a larger HPR. $\mathrm{N}_{\mathrm{vp}}$, which represents the number of corrective attempts made mid-movement, is also a surrogate marker that has been used in publications. ${ }^{(4,5)}$

\footnotetext{
${ }^{1}$ Department of Rehabilitation Medicine, Singapore General Hospital, ${ }^{2}$ Duke-NUS Graduate Medical School, ${ }^{3}$ Department of Occupational Therapy, Singapore General Hospital, ${ }^{4}$ Pillar of Information Systems Technology and Design Department, Singapore University of Technology and Design, Singapore

Correspondence: Dr Geoffrey S Samuel, Registrar, Department of Rehabilitation Medicine, Singapore General Hospital, Academia Building Level 4, Outram Road, Singapore 169608. Geoffrey.sithamparapillai.samuel@sgh.com.sg
} 
Before and after the course of VR-based therapy sessions, the patient was assessed using the following clinical assessments: (a) Functional Independence Measure (FIM), an assessment of ability to perform 13 different activities of daily living and five cognitive domains. For each item measured, a score of 6-7 indicates functional independence, 5 denotes supervision/ contact assistance, 4 minimal assistance, 3 moderate assistance, 2 maximal assistance and 1 complete dependence; (b) UpperExtremity Fugl-Meyer (FM) Scale, an assessment of upper limb tone, power and movements; and (c) the Action Research Arm Test (ARAT), an assessment tool for upper limb grasp, grip, pinch and gross movements. These established assessments for poststroke patients are widely used; however, floor and ceiling effects have been reported. ${ }^{(6)}$ The assessments were made by an experienced occupational therapist blinded to the patient's day-to-day therapy.

The patient showed good progress in her general recovery. Her total length of stay was 13 days, with nine days spent in rehabilitation. (Two weeks of inpatient rehabilitation had been planned; however, she was discharged early due to personal reasons.) The patient participated in seven out of ten sessions of VR-based therapy. Each session lasted 20 minutes and contained an average of seven minutes of active therapy and an estimated 97 repetitions of flexion-extension of the right elbow. At the end of the course of therapy, the motor power of the patient's right upper limb improved to 4.5/5 on clinical assessment. Her FIM scores improved from 87 (out of a maximum score of 126) prior to the sessions to 113 after the last session. The results suggest that the patient improved from needing minimal assistance for activities of daily living to functional independence. Correspondingly, her FM scores improved from 56/66 to $62 / 66$, with the most improvement in speed and coordination of movements of the affected upper limb. This correlated with a clinically significant improvement in upper limb speed and quality of movement. ARAT scores showed improvements in gross arm movements such as placing the hand or on top of the head and bringing the hand to the mouth, although the overall change in ARAT score was not clinically significant. The patient's scores on the three types of clinical assessments are summarised in Table I.

Kinematic parameters derived from IMU output on joint position showed similar results as the clinical assessments. The HPR and $\mathrm{N}_{\mathrm{vp}}$ of the patient's affected hand improved significantly over the course of seven sessions (Figs. 2 \& 3). Her unaffected hand was used as a comparator. Time taken to perform each flexion movement was also recorded. The patient made vast improvements in the speed at which she completed each target movement (Fig. 4).

In response to surveys before and after the course of VR-based therapy, the patient commented that she generally found the VR-based therapy engaging and stimulating. She perceived her participation in this additional therapy as beneficial and was keen to continue similar VR-based therapy after her discharge. The patient reported no adverse outcomes from participating in the additional VR-based therapy.
Table I. Summary of the patient's FIM, FM motor subscores and ARAT subscores.

\begin{tabular}{|c|c|c|}
\hline Item assessed & $\begin{array}{c}\text { On } \\
\text { admission }\end{array}$ & $\begin{array}{l}\text { On } \\
\text { discharge }\end{array}$ \\
\hline FIM & 87 & 113 \\
\hline Eating & 5 & 7 \\
\hline Grooming & 5 & 7 \\
\hline Dressing upper body & 3 & 6 \\
\hline Dressing lower body & 4 & 7 \\
\hline Bathing & 3 & 6 \\
\hline Toileting & 4 & 7 \\
\hline Bladder & 7 & 7 \\
\hline Bowel & 7 & 7 \\
\hline Transfer: bed/chair & 4 & 5 \\
\hline Transfer: toilet & 4 & 5 \\
\hline Transfer: shower & 4 & 5 \\
\hline Ambulation* & 2 & 5 \\
\hline Stair-climbing & 1 & 5 \\
\hline Comprehension & 7 & 7 \\
\hline Expression & 7 & 7 \\
\hline Social interaction & 7 & 7 \\
\hline Memory & 7 & 7 \\
\hline Problem-solving & 6 & 6 \\
\hline ARAT subscore (affected arm) & 41 & 45 \\
\hline Grasp $(\max =18)$ & 14 & 12 \\
\hline Grip (max = 12) & 9 & 12 \\
\hline Pinch $(\max =18)$ & 12 & 12 \\
\hline Gross movements ( $\max =9$ ) & 6 & 9 \\
\hline $\begin{array}{l}\text { FM assessment motor subscore } \\
\text { (affected arm) }\end{array}$ & 56 & 62 \\
\hline Upper extremity ( $\max =36)$ & 32 & 33 \\
\hline Wrist $(\max =10)$ & 9 & 9 \\
\hline Hand $(\max =14)$ & 13 & 14 \\
\hline Coordination/speed subscore $(\max =6)$ & 2 & 6 \\
\hline
\end{tabular}

In general, the closer the score to the maximum, the better the patient can perform the task. *Ambulated $50 \mathrm{~m}$ and $150 \mathrm{~m}$ on admission and on discharge, respectively. ARAT: Action Research Arm Test; FIM: Functional Independence Measure; FM: Fugl-Meyer

\section{DISCUSSION}

Neuroplasticity is the structural and functional adaptation of the nervous system to a change in the environment through a consequent change in behaviour. ${ }^{(7)} \mathrm{VR}$-based therapy facilitates the process of neuroplasticity by creating a stimulating environment favourable to the development of increased and efficacious synaptic connections. It does so through producing high-intensity, active, repetitive and task-oriented movements, utilising the principles of motor learning. ${ }^{(8)}$

A 2011 Cochrane article on the use of VR-based therapy in stroke recovery, which reviewed 19 randomised controlled trials (with a total of 565 poststroke participants), concluded that there was some evidence that VR-based therapy was more effective than conventional interventions and achieved better arm function. ${ }^{(1)}$ The review also indicated that patients generally tolerated VR-based therapy well with few and mild adverse events. Many of the studies reviewed were done in an outpatient 


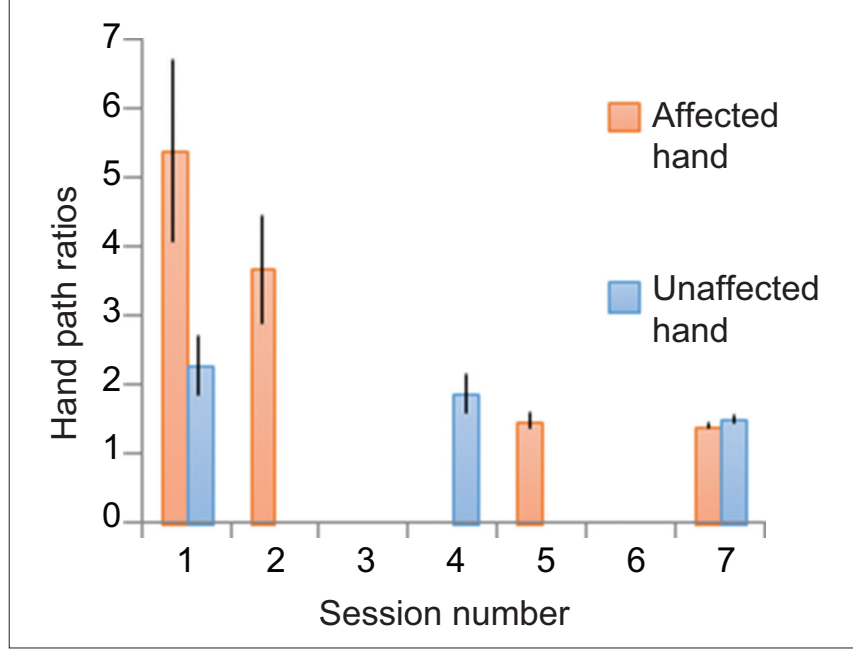

Fig. 2 Bar chart shows improvement in hand path ratios for movement smoothness over seven sessions. Data for the third and sixth sessions was not captured by the devices.

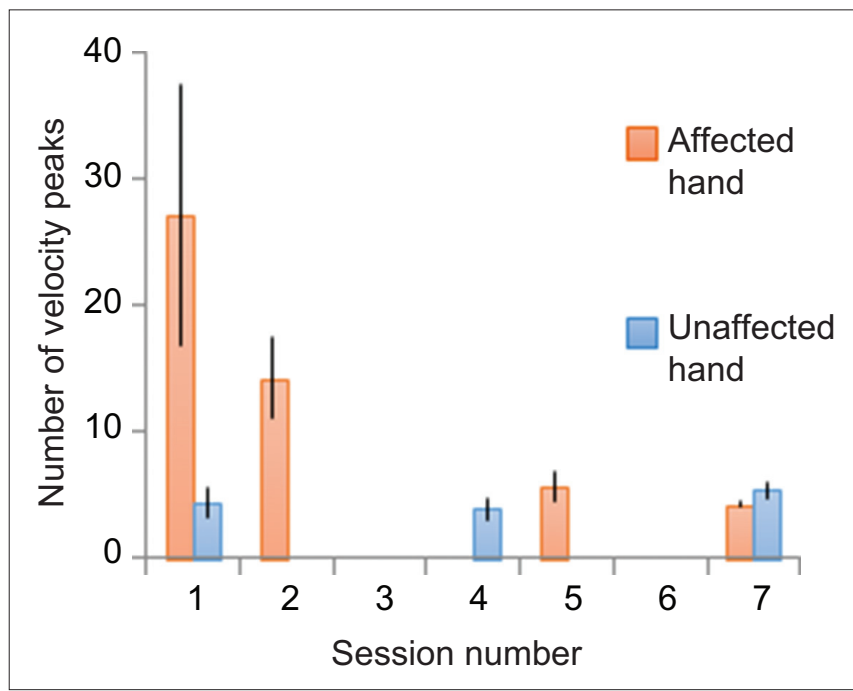

Fig. 3 Bar chart shows improvement in number of velocity peaks for movement smoothness over seven sessions. Data for the third and sixth sessions was not captured by the devices.

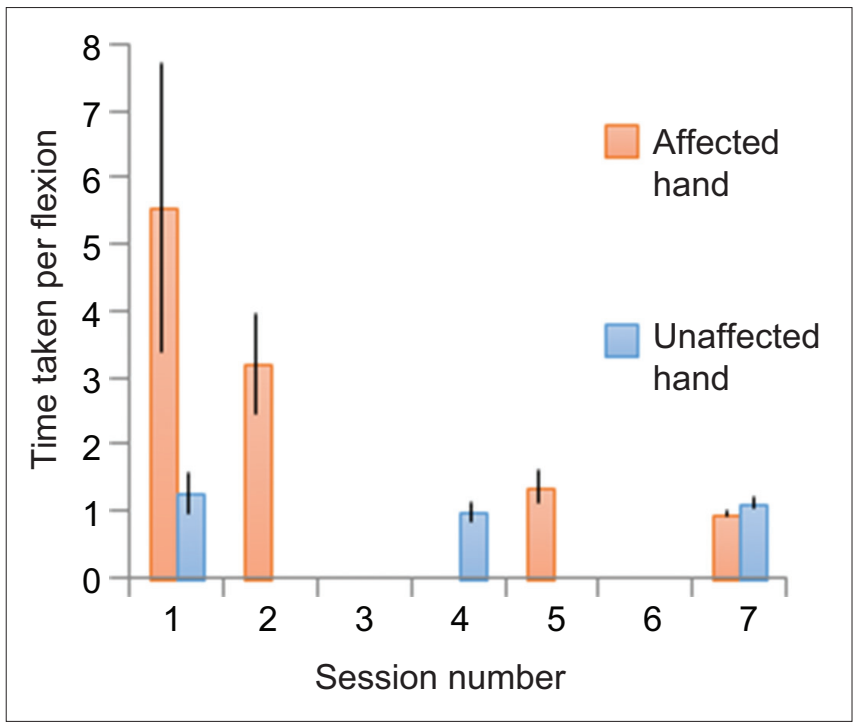

Fig. 4 Bar chart shows improvement in flexion-extension of the affected arm over seven sessions. Data for the third and sixth sessions was not captured by the devices. setting during the chronic phase of stroke recovery (more than six months from initial symptoms). The total number of hours of therapy provided per study ranged from less than 5 hours of total therapy to more than 21 hours. A variety of VR-based therapies were used, both commercial and specially developed as well as immersive and non-immersive. ${ }^{(1)}$

Our project was novel in its integration of VR-based therapy during the acute phase of stroke recovery (within two weeks of onset of symptoms) in order to take maximal advantage of the prime period for motor recovery. ${ }^{(7)}$ Our patient generally found the VR program easy to use although she had only primary level education and no prior experience with computer games. This suggests that with appropriate program and patient selection, patients can be effectively engaged by VR-based therapy. For sufficiently motivated and technologically adept patients, VR-based therapy may be a good option for self-directed therapy beyond the standard therapy provided by allied health professionals.

The use of kinematic assessments to track the patient's movements and video recording technology with immediate playback facilitated specific and quantitative feedback to the patient on her performance and improvement. Kinematic analysis has been increasingly used as an outcome measure in recent research due to its sensitivity, reliability and objectivity in comparison to ordinal clinical measurement scales. ${ }^{(9,10)}$ Sensitivity of measurements to improvement is especially important as a source of motivation for patients, as rehabilitation is usually an ongoing process and clinical improvements are sometimes only apparent after a number of weeks.

One possible confounder in this study was the difficulty of differentiating the improvements in the patient's spontaneous recovery from the benefits of the standard physiotherapy and occupational therapy that she received. Standard poststroke occupational therapy and physiotherapy involve strengthening, stretching, balance, gait training, training in functional tasks and caregiver training. It was also likely that the patient suffered an acute lacunar infarct and thus had high potential for recovery from stroke. Nonetheless, her case highlights the feasibility of taking such technology into the wards as part of a course of acute inpatient rehabilitation.

Our department is also conducting further research on combining VR-based therapy with neuropharmacological intervention for acute stroke rehabilitation. This corresponds with the thrust of related research by the international community into determining the optimal type, timing, setting and duration of VR-based therapy.

In conclusion, VR-based therapy shows great promise as an adjunctive therapy to rehabilitation in upper limb stroke recovery and can effectively be used in an acute inpatient setting. Its contribution to the rehabilitation process includes improved patient motivation and engagement, immediate quantitative feedback and possibly more sensitive tracking of patients' recovery. Further research needs to be carried out to determine the most efficacious type, timing, setting and duration of VRbased therapy. 


\section{ACKNOWLEDGEMENTS}

The authors wish to acknowledge that this case study is part of an ongoing research initiative funded by the SingHealth Medicine Academic Clinical Program under the 'Pitch for Funds' grant. The VR game component is supported by MultiplAtform Game Innovation Centre (MAGIC), funded by the Singapore National Research Foundation under its IDM Futures Funding Initiative and administered by the Interactive Digital Media Programme Office, Media Development Authority. Special thanks to Singapore University of Technology and Design programmers Tom Kueh and Raina Lim, as well as students Immanuella Lim and Clarence Teo, who helped develop the VR game programme.

\section{REFERENCES}

1. Laver KE, George S, Thomas S, Deutsch JE, Crotty M. Virtual reality for stroke rehabilitation. Cochrane Database Syst Rev 2011; (9):CD008349.

2. Venketasubramanian N, Chen CL. Burden of stroke in Singapore. Int J
Stroke 2008; 3:51-4

3. Broeren J, Sunnerhagen KS, Rydmark M. A kinematic analysis of a haptic handheld stylus in a virtual environment: a study in healthy subjects. J Neuroeng Rehabil 2007; 4:13.

4. Holden MK, Dyar TA, Schwamm L, Bizzi E. Virtual-environment-based telerehabilitation in patients with stroke. Presence 2005; 14:214-33.

5. Caimmi M, Carda S, Giovanzana C, et al. Using kinematic analysis to evaluate constraint-induced movement therapy in chronic stroke patients. Neurorehabil Neural Repair 2008; 22:31-9.

6. Lin JH, Hsu MJ, Sheu CF, et al. Psychometric comparisons of 4 measures for assessing upper-extremity function in people with stroke. Phys Ther 2009; 89:840-50.

7. Rossini PM, Calautti C, Pauri F, Baron JC. Post-stroke plastic reorganisation in the adult brain. Lancet Neurol 2003; 2:493-502.

8. Ng YS, Chew E, Samuel GS, Tan YL, Kong KH. Advances in rehabilitation medicine. Singapore Med J 2013; 54:538-51.

9. Alt Murphy M, Willén C, Sunnerhagen KS. Responsiveness of upper extremity kinematic measures and clinical improvement during the first three months after stroke. Neurorehabil Neural Repair 2013; 27:844-53.

10. van Dokkum L, Hauret I, Mottet D, et al. The contribution of kinematics in the assessment of upper limb motor recovery early after stroke. Neurorehabil Neural Repair 2013; 28:4-12. 